\title{
Glycated albumin is an optimal biomarker for gestational diabetes mellitus
}

\author{
YAPING HUANG, YONGWEI HU, YU MA and GUANGYONG YE \\ Women's Hospital, School of Medicine, Zhejiang University, Hangzhou, Zhejiang 310006, P.R. China
}

Received August 3, 2015; Accepted October 15, 2015

DOI: $10.3892 /$ etm.2015.2808

\begin{abstract}
Gestational diabetes mellitus (GDM) refers to abnormal glucose tolerance, which is a common complication that occurs in some women for the first time during the gestation period. However, the relationship between onset of GDM and factors including advanced age and a family history of diabetes remains to be determined. The study aimed to examine the clinical significance of the detection of glycated albumin (GA) in pregnant women with GDM. A total of 893 cases of pregnant women with GDM were included, with 661 healthy pregnant women serving as the normal controls. A conditional logistic regression model was used to analyze the univariate and multivariate data to estimate the odds ratio (OR) and $95 \%$ confidence interval $(95 \% \mathrm{CI})$. As the gestational weeks increased, the fasting blood glucose (FGP) concentration and GA-L value of the pregnant women in the normal control group gradually decreased whereas those of pregnant women with GDM greatly increased. The univariate analysis revealed that the impact factors on the occurrence of early-onset neonatal sepsis included, mother's age $>35$ years, complication of pregnancy hypertension, family history of hypertension, family history of diabetes, cesarean delivery, height, BMI, GA-L, and FGP. The multivariate logistic regression analysis revealed that the complication of pregnancy hypertension $(\mathrm{OR}=3.302 ; 95 \% \mathrm{CI}$, 1.705-6.394), family history of hypertension ( $\mathrm{OR}=2.970$; 95\% CI, 1.520-5.801), GA-L (OR=1.556; 95\% CI, 0.940-2.012) and FGP $(\mathrm{OR}=5.431 ; 95 \% \mathrm{CI}, 4.097-7.198)$ were the main factors for pregnant women with GDM. In conclusion, pregnant women with GDM may be affected by various factors. Additionally, GA may be applied to reflect the recent blood glucose control on pregnant women with GDM.
\end{abstract}

\section{Introduction}

Gestational diabetes mellitus (GDM) refers to abnormal glucose tolerance, a common complication that occurs in some women

Correspondence to: Dr Guangyong Ye, Women's Hospital, School of Medicine, Zhejiang University, 1 Xueshi Road, Shangcheng, Hangzhou, Zhejiang 310006, P. R. China

E-mail:ygy20518158@sina.com

Key words: gestational diabetes mellitus, glycated albumin, factors for the first time during the gestation period (1). According to the latest diagnostic criteria of the International Association of Diabetes and Pregnancy Study Groups (IADPSG), the incidence of GDM has increased to $17.8 \%$ (2). GDM may lead to many complications in mothers and infants and pose a serious threat to their health. The short- and long-term effects of GDM on pregnant and lying-in women and perinatal infants have aroused considerable attention (2-4).

Early diagnosis and the timely treatment of GDM have always been a focus of clinicians. In recent years, clinical studies have shown that the onset of GDM was closely associated with a number of factors, including gestational hypertension, polyhydramnios, infection, advanced age and family history of diabetes and obesity in pregnancy (3-5). Further clinical investigation is required to verify the precise relationship between the onset of GDM and some of the above influencing factors in order to make a clear diagnosis and identify treatment for this disease.

At present, the treatment of GDM is mainly focused on the monitoring, evaluation and control of blood sugar. In clinic, two types of indicators are monitored, the instant blood glucose (such as fasting blood glucose) and the long-term blood glucose monitoring indicator, such as glycosylated hemoglobin (HbAlc). However, the two indicators have their shortcomings. Fasting blood glucose is greatly influenced by previous diet, mental state and other factors such as stress, and exhibits great fluctuations, making it difficult to control. HbAlc only reflects the blood glucose level during the previous 2-3 months and has a relatively shorter observation period for GDM, thus, it is not sensitive. Glycated albumin (GA) reflects the average blood glucose within 2-3 weeks and also provides a short-term monitoring indicator. Additionally, the level of GA was not affected by the red blood cell lifetime. The level of GA has been utilized to monitor the status of blood glucose control in diabetics. However, few reports besides Asian studies are currently available on the relationship between GA and the occurrence of GDM and blood glucose control.

\section{Materials and methods}

Selection of cases. Pregnant and lying-in women with GDM who had undergone childbirth in the Women's Hospital, School of Medicine, Zhejiang University (Zhejiang, China), between August 2013 and May 2014 were selected for this study. Additionally, pregnant women without GDM were selected as normal controls. The study was approved by the Institutional 
Ethics Committee of Women's Hospital, School of Medicine, Zhejiang University (Zhejian, China). Written informed consent was obtained from each participant. The diagnostic criteria as stipulated by IADPSG, included a $75 \mathrm{~g}$ glucose tolerance test and fasting blood glucose (FGP; after a 12 -h fast).

Specific diagnostic criteria were as follows: i) Oral glucose tolerance test (OGTT; $75 \mathrm{~g}$ ), for which the subjects were required to fast for 8-14 $\mathrm{h}$ prior to the test, and received $300 \mathrm{ml}$ liquid composed of $75 \mathrm{~g}$ glucose orally in $5 \mathrm{~min}$. Elbow venous blood $(3 \mathrm{ml})$ was withdrawn during the fasting state and at 1 and $2 \mathrm{~h}$ after glucose load to measure the blood glucose, using the glucose oxidase method (Glucose Kit; BioSino Bio-Technology and Science Inc., Beijing, China). ii) IADPSG criteria: If one or more of the following criteria were exceeded during the OGTT, a diagnosis of GDM was considered. Blood glucose levels were measured at fasting, $5.1 \mathrm{mmol} / \mathrm{l}$; at $1 \mathrm{~h}$, $10.0 \mathrm{mmol} / \mathrm{l}$; and at $2 \mathrm{~h}, 8.5 \mathrm{mmol} / \mathrm{l}$. If fasting blood glucose was $\geq 7.0 \mathrm{mmol} / 1$ or HbAlc $\geq 6.5 \%$ or random blood glucose was $\geq 11.1 \mathrm{mmol} / 1$, the diagnosis was overt diabetes mellitus.

Determination of influencing factors. An influencing factor record (questionnaire) for pregnant and lying-in women with gestational diabetes mellitus was created. Information of the aforementioned and normal control cases, including mother's age, mother's degree of education, urban resident or not, cesarean delivery, BMI, weight, height, hypertensive disorders complicating pregnancy (HDP), in vitro fertilization (IVF), preterm birth, GA-L, FGP, family hypertension history, and family diabetes history was recorded.

Detection method of GA-L and FGP. Detection of GA was carried out using the Lucica GA-L glycated albumin assay kit (Lucica ${ }^{\circledR}$ GA-L; Asahi Kasei Corp., Tokyo, Japan), which utilizes bromcresol purple, and has higher specificity for albumin, as compared to the previously used bromocresol green, which was not as specific. The levels of GA and serum albumin (GA-L) were expressed as a percentage to exclude the influence of serum albumin on the detection result. Blood glucose was measured using the glucose oxidase method.

Definition and assignment of major variables. For dependent variables GD cases were assigned as 1 and control subjects were assigned 0 (Table I). Definitions of some of the independent variables were as follows: Elderly parturient women, age $>35$ years; BMI, weight in kilograms divided by the square of height in meters; IVF, in vitro fertilization; hypertensive disorders complicating pregnancy (HDP) were considered as a disease characterized by the coexistence of pregnancy and hypertension (systolic pressure $\geq 140 \mathrm{mmHg}$ and/or diastolic pressure $\geq 90 \mathrm{mmHg}$ ) or with urinary protein $\geq 0.3 \mathrm{~g} / 24 \mathrm{~h}$, or positive random urinary protein after pregnancy for 20 weeks, and in severe cases, with convulsion; preterm birth was considered as the birth of a baby at $>28$ weeks and $<37$ weeks gestational age; family hypertension history included one or several relatives with hypertension; and family diabetic history included one or several relatives with diabetes (Fig. 1).

Statistical analysis. EpiData 3.1 (The EpiData Association, Odense, Denmark) was used to construct the database with the original data, and then the data were transformed into a
Table I. Assignment table of some influencing factors of GDM pregnant women.

\begin{tabular}{lcc} 
Indicator & & Assignment \\
\hline Mother's age $>35$ years & Yes & 1 \\
& No & 0 \\
Permanent job & Yes & 1 \\
& No & 0 \\
Pregnancy hypertension & Yes & 1 \\
& No & 0 \\
HDP & Yes & 1 \\
& No & 0 \\
Family diabetic history & Yes & 1 \\
& No & 0 \\
College education & Yes & 1 \\
& No & 0 \\
External fertilization & Yes & 1 \\
& No & 0 \\
Preterm birth & Yes & 1 \\
& No & 0 \\
Cesarean delivery & Yes & 1 \\
& No & 0 \\
Weight & Constant argument \\
Height & Constant argument \\
BMI & Constant argument \\
GA-L & Constant argument \\
FGP & Constant argument & \\
&
\end{tabular}

GDM, gestational diabetes mellitus; HDP, hypertensive disorders complicating pregnancy; FGP, fasting blood glucose.

recognizable format. SPSS 16.0 software (IBM Corporation, Chicago, IL, USA) was used for statistical analysis for conducting single and multiple factor conditional logistic regression analysis and to calculate the odds ratio (OR) and 95\% confidence interval (CI). If OR, 95\% CI did not include 1, the result was considered statistically significant.

\section{Results}

General condition. The total number of subjects included in the study was 1,554 , of which 893 pregnant women were confirmed with GDM and 661 healthy pregnant women served as the normal controls. The median pregnancy weeks of the GDM cases was 26 weeks and gestational weeks with a relatively higher diagnostic rate were 24-26 weeks (diagnostic rate, 78.22\%), 27-29 weeks (diagnostic rate, 63.57\%), and 30-32 weeks (diagnostic rate, 61.64\%) (Fig. 1).

Changes of FGP concentration and GA-L of pregnant women in different gestational weeks. The FGP concentration and GA-L level in the pregnant women with GDM at different gestational weeks were relatively higher compared to the pregnant women in the normal control group. As the gestational weeks increased, the FGP concentration and GA-L value of the pregnant women in the normal control group gradually 


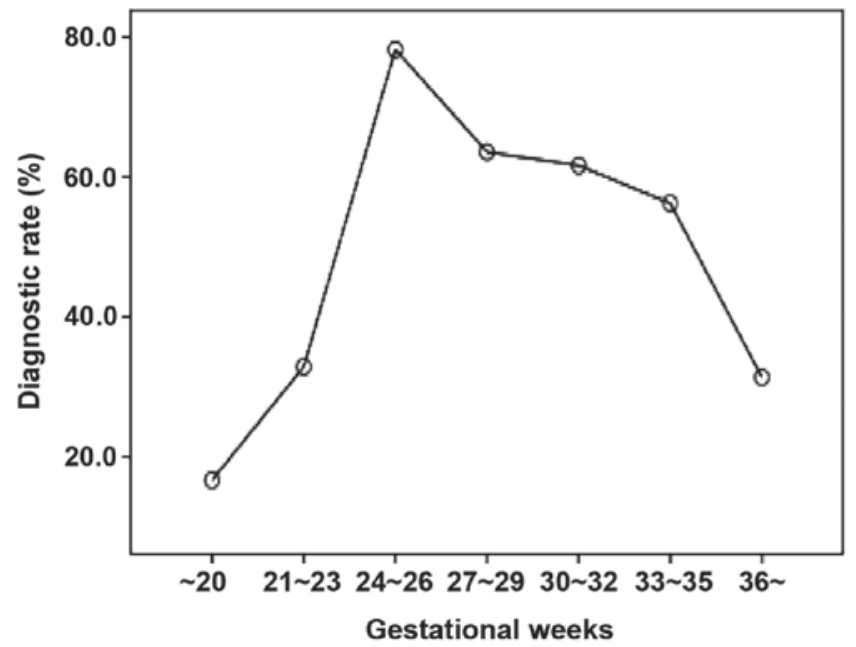

Figure 1. Diagnostic rate of GDM in different pregnancy phases. The median pregnancy weeks of the GDM cases was 26 weeks. Gestational weeks with a relatively higher diagnostic rate were 24-26 weeks (diagnostic rate, $78.22 \%$ ), 27-29 weeks (diagnostic rate, 63.57\%), 30-32 weeks (diagnostic rate, 61.64\%). GDM, gestational diabetes mellitus.

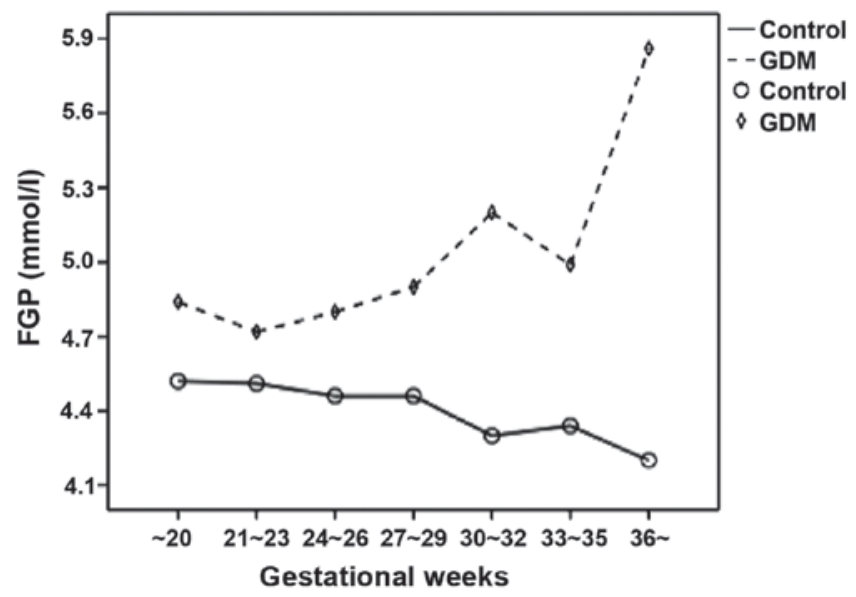

Figure 2. Changes in the FGP concentration in different pregnancy phases. Compared to the normal control pregnant women, the pregnant women with GDM had a relatively higher FGP concentration at different gestational weeks. As the gestational weeks increased, the FGP concentration of pregnant women in the normal control group gradually decreased while those of the pregnant women with GDM markedly increased. FGP, fasting blood-glucose; GDM, gestational diabetes mellitus.

decreased while those of the pregnant women with GDM markedly increased (Figs. 2 and 3). The results showed that FGP concentration and GA-L value in all pregnant women were significantly correlated $\left(\mathrm{P}=0.000 ; \mathrm{R}^{2}=0.103\right)$ (Fig. 4).

Single factor analysis of the general condition of puerperas. The result of the single factor analysis (Table II) revealed that factors that were statistically different with the occurrence of GDM included: Mother's age $>35$ years $(\mathrm{OR}=1.802$; 95\% CI, 1.306-2.487), complication of pregnancy hypertension $(\mathrm{OR}=3.381 ; 95 \% \mathrm{CI}, 1.910-5.983)$, family hypertension history $(\mathrm{OR}=1.678 ; 95 \% \mathrm{CI}, 1.132-2.486)$, family diabetic history (OR=3.848; 95\% CI, 2.101-7.047), cesarean delivery $(\mathrm{OR}=1.544 ; 95 \% \mathrm{CI}, 1.261-1.891)$, height $(\mathrm{OR}=0.033 ; 95 \% \mathrm{CI}$, 0.004-0.307), BMI (OR=1.039; 95\% CI, 1.006-1.073),

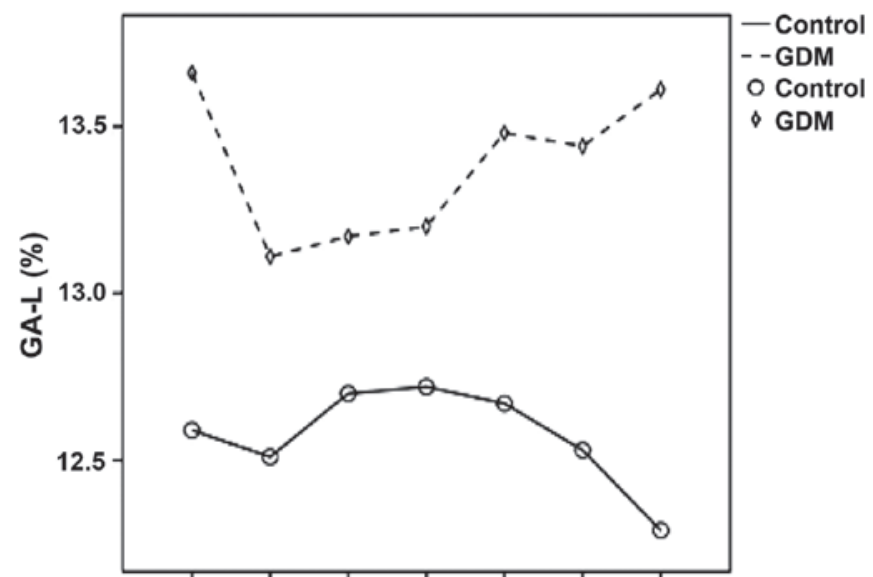

$\sim 20 \quad 21 \sim 23 \quad 24 \sim 26 \quad 27 \sim 29 \quad 30 \sim 32 \quad 33 \sim 35 \quad 36 \sim$ Gestational weeks

Figure 3. Change of GA-L levels in different phases of pregnancy. Compared to the pregnant women in the normal control group, a higher GA-L level was observed in the pregnant women with GDM at different gestational weeks. As the gestational weeks increased, the GA-L value of the pregnant women in the normal control group gradually decreased while that of the pregnant women with GDM greatly increased. GDM, gestational diabetes mellitus.

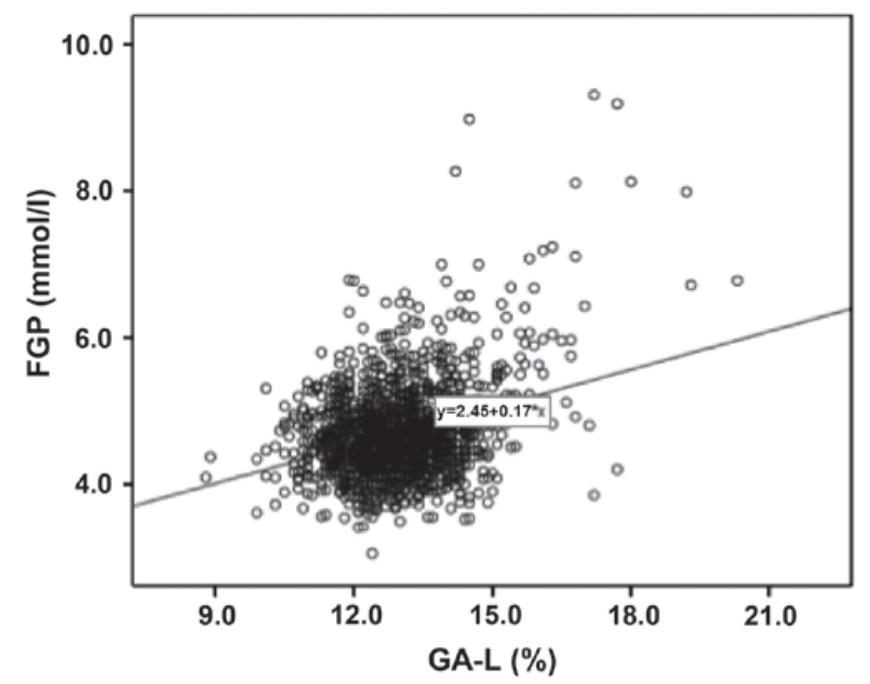

Figure 4. Relationship between the FGP and GA-L concentration in pregnant women. The FGP concentration and GA-L value in all pregnant women exhibited a significant correlation $\left(\mathrm{R}^{2}=0.103\right)$. FGP, fasting blood-glucose.

GAL (OR=1.637; 95\% CI, 1.479-1.813), and FGP (OR=5.460; 95\% CI, 4.246-7.020).

Factors that had no direct correlation with GDM included: College education (OR=0.965; 95\% CI, 0.736-1.265), external fertilization $(\mathrm{OR}=1.494 ; 95 \% \mathrm{CI}, 0.741-3.009)$, preterm birth $(\mathrm{OR}=1.006$; $95 \% \mathrm{CI}, 0.657-1.541)$, and weight $(\mathrm{OR}=1.005$; 95\% CI, 0.994-1.017).

Multiple conditional logistic regression analysis. The variables with statistical significance from the single factor analysis were considered as independent variables, and included, mother's age $>35$ years, complication of pregnancy hypertension, family hypertension history, family diabetic history, cesarean delivery, height, BMI, GA-L, and FGP, respectively. Following 
Table II. Single factor analysis of GDM.

\begin{tabular}{lcc}
\hline Factor & P-value & OR (95\% CI) \\
\hline $\begin{array}{l}\text { Mother's age } \\
>35 \text { years }\end{array}$ & \\
HDP & 0.000 & $1.802(1.306-2.487)$ \\
FHH & 0.000 & $3.381(1.910-5.983)$ \\
FDH & 0.010 & $1.678(1.132-2.486)$ \\
College education & 0.000 & $3.848(2.101-7.047)$ \\
External fertilization & 0.795 & $0.965(0.736-1.265)$ \\
Preterm birth & 0.262 & $1.494(0.741-3.009)$ \\
Cesarean delivery & 0.977 & $1.006(0.657-1.541)$ \\
Weight & 0.000 & $1.544(1.261-1.891)$ \\
Height & 0.372 & $1.005(0.994-1.017)$ \\
BMI & 0.003 & $0.033(0.004-0.307)$ \\
GA-L & 0.019 & $1.039(1.006-1.073)$ \\
FGP & 0.000 & $1.637(1.479-1.813)$ \\
\end{tabular}

GDM, gestational diabetes mellitus; OR, odds ratio; CI, confidence interval; HDP, hypertensive disorders complicating pregnancy; $\mathrm{FHH}$, family hypertension history; FDH, family diabetic historyFGP, fasting blood-glucose.

the results of the multiple conditional logistic regression analysis occurrence and non-occurrence of GDM were considered as dependent variables. The multiple conditional logistic regression model was employed to analyze the results from the complication of pregnancy hypertension $(\mathrm{OR}=3.302 ; 95 \% \mathrm{CI}$, 1.705-6.394), family hypertension history (OR=2.970; 95\% CI, 1.520-5.801), GA-L (OR=1.556; 95\% CI, 0.940-2.012) and FGP (OR=5.431; 95\% CI, 4.097-7.198) (Table III and Fig. 3).

\section{Discussion}

GDM poses a serious threat to mothers and infants (6). GDM may be controlled using a series of treatments, including kinesitherapy, diet control, and insulin. Other methods, such as strengthening the monitoring and caring of pregnant mothers and infants, and selecting appropriate delivery methods and delivery days based on the condition of pregnant mothers, may decrease the complications of mothers and infants and improve the prognosis of perinatal infants. Therefore, a timely diagnosis of diabetes is crucial. Diagnostic criteria of GDM have been unified, and include NDDG, ADA and IADPSG criteria (7). In China, such criteria remain to be established. Since 2011, our hospital has begun to utilize IADPSG criteria as diagnostic criteria of GDM. IADPSG criteria do not include the data of patients from the Chinese mainland, which accounts for a large proportion of the overall Asian population. The time period for GDM screening and diagnosis in Chinese pregnant women was investigated in the present study. Our results indicated that 24-29 weeks was the most appropriate time period to screen the pregnant women with GDM.

The cause of GDM is different to that of general diabetes. WHO defined GDM as an independent diabetes type that is different from type 1 and type 2 diabetes. GDM is regarded as an endocrine and metabolic disease characterized by hereditary inclination and induced by various factors $(5,8-10)$. Similar to general type 2 diabetes, gestational diabetes has numerous common risk factors, and specific causes of diseases associated with pregnancy. In a previous study (10), a multiple factor analysis was conducted and the results revealed that GA-L, FGP, HDP and family diabetic history were closely associated with the onset of GDM.

GDM may result in the elevation of the blood glucose levels of patients, causing damage to mothers and infants. After the insulin resistance and insulin secretion function of GDM patients was impaired, their insulin secretion peak exhibited a delay, eventually leading to hyperglycemia. Hyperglycemia subsequently resulted in numerous short-term effects, including the complication of early preeclampsia in pregnant women with GDM, the increasing incidence of abortion, preterm birth and cesarean delivery, the significant increase of fetal malformation, fetal macrosomia, birth trauma, neonatal hypoglycemia and other diseases. Long-term effects included a higher risk of type 2 diabetes on puerperas themselves and a higher risk of obesity in adolescence, as well as hypertension and diabetes in adulthood of their offspring $(2-4,10,11)$. Adverse pregnancy outcomes appeared to be closely associated with the blood glucose. The results of the present study have shown that the concentration of FGP and value of GA-L in pregnant women with GDM at different gestational weeks increased. The FGP concentration and GA-L value of the pregnant women with GDM increased, indicating that the glucose metabolic pathways of pregnant women with GDM underwent a form of decompensation. In pancreatic $\beta$-cells, increased $\beta$-cell volume and increased insulin secretion reaction occurs to compensate for their insulin resistance during pregnancy. It is considered that abnormal glucose tolerance develops in pregnant women when

Table III. Multiple conditional logistic regression analysis of risk factors of GDM.

\begin{tabular}{|c|c|c|c|c|c|}
\hline Factor & Regression coefficient & Standard error & Wald & P-value & OR $(95 \% \mathrm{CI})$ \\
\hline HDP & 1.194 & 0.337 & 12.546 & 0.000 & $3.302(1.705-6.394)$ \\
\hline Family diabetic history & 1.088 & 0.342 & 1.103 & 0.001 & $2.970(1.520-5.801)$ \\
\hline FGP & 1.692 & 0.144 & 138.519 & 0.000 & $5.431(4.097-7.198)$ \\
\hline GA-L & 0.442 & 0.061 & 52.664 & 0.000 & $1.556(0.940-2.012)$ \\
\hline
\end{tabular}

GDM, gestational diabetes mellitus; OR, odds ratio; CI, confidence interval; HDP, hypertensive disorders complicating pregnancy; FGP, fasting blood-glucose. 
this compensatory effect is insufficient. The presence of pancreatic $\beta$-cell dysfunction in GDM has been demonstrated (12).

GA reflects the recent blood glucose control state of pregnant women with GDM. In the studies conducted by Yoshiuchi et al (13), it was confirmed that GA and the blood glucose in patients with type 1 and type 2 diabetes were closely correlated with $\mathrm{HbAlc}$, with GA being a better indicator for monitoring the blood glucose control of patients with diabetes. According to a report by the Japan Glycated Albumin Study Group of the Japanese Society of Diabetes and Pregnancy, the frequencies of neonatal complications (neonatal hypoglycemia, polycythemia, and respiratory disorder) and babies that are large for gestational age were significantly higher in the group whose GA value at the end of pregnancy was $\geq 15.8 \%$ compared with those of the group whose GA value was $<15.8 \%$ (14). Based on these results, the Japanese Society of Diabetes and Pregnancy strongly recommended that GA measurement was useful for the prevention of perinatal complications in mothers and fetus/infants (15).

Our results demonstrated that GA and the concentration of FGP on pregnant women with and without GDM were closely correlated. The multi-factor regression analysis revealed that the level of GA in pregnant women was closely associated with the condition irrespective of GDM. Since GDM usually occurred during pregnancy and the course was relatively short, FGP and dynamic blood glucose monitoring may have some drawbacks. Additionally, HbAlc may not reflect the short-term change of blood glucose resulting from dietary changes or insulin treatment. Compared with HbAlc, the half-life period of GA was 17-19 days and it was able to reflect the average blood glucose within 2-3 weeks, making it more sensitive to short-term blood glucose change. Therefore, it was suggested that GA be applied to reflect the recent blood glucose control in pregnant women with GDM.

Pregnant women with GDM suffered a higher risk (2- to 4-fold) of experiencing complications by HDP than pregnant women without diabetes. Previous findings (16-18) have identified that GDM and HDP may have a common origin. Severe insulin resistance and hyperinsulinemia were closely associated with the complication of GDM by HDP. During the second and third trimester, placentae secreted various types of insulin antagonists, such as estriol, which reached a peak during the 32-34 weeks, resulting in a gradual increase of insulin resistance. The resulting hyperinsulinemia, in turn, caused the hyperfunction of the sympathetic nervous system, retention of sodium and water, with HDP aggravating the insulin resistance. Inflammatory factor adiponectin was able to adjust the glycerolipid metabolism of the body, protect blood vessel endothelium, and reduce blood pressure. Adiponectin is known to play an important role in the patho-mechanism of GDM and HDP (19).

Family history of diabetes has been associated with the occurrence of GDM. Duman et al (17) studied 650 cases of outpatient pregnant women in Turkey and showed that family diabetic history was a predominant risk that predisposes pregnant women to developing GDM. Additionally, previous studies $(20,21)$ have suggested that the history of diabetes immediate family members and previous GDM history of pregnant women potentially increased the incidence of GDM during gestation. The results of the present study are consistent with findings of the aforementioned studies.

\section{Acknowledgements}

The present study was supported by the Department of Education of Zhejiang Province (N20120047).

\section{References}

1. Yuen L and Wong VW: Gestational diabetes mellitus: Challenges for different ethnic groups. World J Diabetes 6: 1024-1032, 2015.

2. Sacks DA, Hadden DR, Maresh M, Deerochanawong C, Dyer AR, Metzger BE, Lowe LP, Coustan DR, Hod M, Oats JJ, et al; HAPO Study Cooperative Research Group: Frequency of gestational diabetes mellitus at collaborating centers based on IADPSG consensus panel-recommended criteria: The Hyperglycemia and Adverse Pregnancy Outcome (HAPO) Study. Diabetes Care 35: 526-528, 2012.

3. Köck K, Köck F, Klein K, Bancher-Todesca D and Helmer H: Diabetes mellitus and the risk of preterm birth with regard to the risk of spontaneous preterm birth. J Matern Fetal Neonatal Med 23: 1004-1008, 2010.

4. Alfadhli EM: Gestational diabetes mellitus. Saudi Med J 36: 399-406, 2015.

5. World Health Orgnization: Definition diagnosis and classification of diabetes mellitus and its complications. World Health Orgnization, Geneva, 1999.

6. Hartling L, Dryden DM, Guthrie A, Muise M, Vandermeer B, Aktary WM, Pasichnyk D, Seida JC and Donovan L: Screening and diagnosing gestational diabetes mellitus. Evid Rep Technol Assess (Full Rep) 210: 1-327, 2012.

7. Agarwal MM: Gestational diabetes mellitus: An update on the current international diagnostic criteria. World J Diabetes 6: 782-791, 2015.

8. Poomalar GK: Changing trends in management of gestational diabetes mellitus. World J Diabetes 6: 284-295, 2015.

9. Harder T and Plagemann A: A role for gestational diabetes in the excess maternal transmission of type 2 diabetes? Diabetes Care 23: 431-432, 2000.

10. Buchanan TA, Xiang AH and Page KA: Gestational diabetes mellitus: Risks and management during and after pregnancy. Nat Rev Endocrinol 8: 639-649, 2012.

11. Ovesen PG, Jensen DM,Damm P, Rasmussen S and Kesmodel US: Maternal and neonatal outcomes in pregnancies complicated by gestational diabetes. a nation-wide study. J Matern Fetal Neonatal Med 28: 1720-1724, 2015.

12. Hashimoto K and Koga M: Indicators of glycemic control in patients with gestational diabetes mellitus and pregnant women with diabetes mellitus. World J Diabetes 6: 1045-1056, 2015.

13. Yoshiuchi K, Matsuhisa M, Katakami N, Nakatani Y, Sakamoto K, Matsuoka T, Umayahara Y, Kosugi K, Kaneto H, Yamasaki Y, et al: Glycated albumin is a better indicator for glucose excursion than glycated hemoglobin in type 1 and type 2 diabetes. Endocr J 55: 503-507, 2008.

14. Shimizu I, Hiramatsu Y, Omori Y and Nakabayashi M: Glycated albumin reflects maternal and perinatal outcome in a multicenter study in Japan. Diabetes and Pregnancy 10: 27-31, 2010.

15. Koga M: Glycated albumin; clinical usefulness. Clin Chim Acta 433: 96-104, 2014.

16. Gordin D, Forsblom C, Groop PH, Teramo K and Kaaja R: Risk factors of hypertensive pregnancies in women with diabetes and the influence on their future life. Ann Med 46: 498-502, 2014.

17. Duman NB: Frequency of gestational diabetes mellitus and the associated risk factors. Pak J Med Sci 31: 194-197, 2015.

18. Pan J, Zhang F, Zhang L, Bao Y, Tao M and Jia W: Influence of insulin sensitivity and secretion on glycated albumin and hemoglobin A1c in pregnant women with gestational diabetes mellitus. Int J Gynaecol Obstet 121: 252-256, 2013.

19. Miehle K, Stepan H and Fasshauer M: Leptin, adiponectin and other adipokines in gestational diabetes mellitus and pre-eclampsia. Clin Endocrinol (Oxf) 76: 2-11, 2012.

20. Chan LY, Wong SF and Ho LC: Diabetic family history is an isolated risk factor for gestational diabetes after 30 years of age. Acta Obstet Gynecol Scand 81: 115-117, 2002.

21. Neelakandan R and Sethu PS: Early universal screening for gestational diabetes mellitus. J Clin Diagn Res 8: OC12-OC14, 2014. 